\title{
Strategies for being random
}

\author{
RONALD A. FINKE \\ University of California, Davis, California
}

Subjects were instructed to generate a string of digits using the numbers $1,2,3$, and 4, either as randomly as possible or as unpredictably as possible. Those subjects who tried to generate the numbers in an unpredictable order produced significantly more consecutive repetitions of the same number than did those who tried to generate the numbers in a random order, and more closely approached the frequency of number repetitions expected by chance. This finding suggests that randomness in behavior may be better achieved by using strategies that avoid the common misconception that, in a truly random sequence of events, successive repetitions ought not to occur.

As a rule, when people attempt to think or behave randomly, they are very poor at doing so. For example, a number of experiments have shown that when subjects are asked to generate a string of digits in as random an order as possible, they exhibit marked departures from what would constitute a purely random sequence (see Wagenaar, 1972, for a general review). When the number set used in the generation of these sequences is fairly small, the most obvious manner in which subjects depart from randomness is that they tend to repeat numbers less often than would be expected by chance (see also Gardner, 1981). One explanation for why repetitions of the same number are avoided in these cases is that people generally believe that such repetitions are not representative of a true random sequence (Kahneman \& Tversky, 1972). However, since a certain frequency of number repetitions should occur even in a random sequence, avoiding such repetitions results in the generation of sequences that are actually less random and more predictable. ${ }^{1}$

Hence, at least one reason why people are not very successful when attempting to generate numbers randomly is that their concept of what constitutes randomness is in error. This suggests that better approximations to randomness might be obtained if, in such experiments, the emphasis were placed, not on being "random," but, rather, on trying to be "unpredictable." The present study compared number sequences generated by subjects who were instructed to try to be as random as possible with sequences generated by subjects instructed to try to fool someone who might attempt to predict which number they would select

This research was supported by a Faculty Research Grant from the University of California, Davis, California. I thank Joel Johnson for helpful comments and suggestions. Requests for reprints should be addressed to: Ronald A. Finke, Department of Psychology, State University of New York, Stony Brook, New York 11794. next. The results provide evidence that the latter strategy leads to the generation of a greater frequency of number repetitions and thus more closely approaches outcomes that would be expected by chance.

\section{METHOD}

\section{Subjects}

Fifty undergraduate students at the University of California at Davis participated as volunteer subjects; they received credit in an introductory psychology course.

\section{Procedure}

The subjects were tested in two groups of 25 each. One group participated in a random condition, and the other participated in a strategy condition. The subjects in the random condition were told that their task was to use the digits $1,2,3$, and 4 to produce a number string that was 50 numbers long and that was in as random an order as possible. In this case, the emphasis was placed on trying to make a purely random sequence composed of these 4 numbers. They were instructed to mark down the numbers they selected by darkening in the appropriate space on a computer scoring card that contained 50 rows of spaces corresponding to each of the 4 numbers. The subjects were further instructed to cover their marks with a blank card after each selection, so that they would not be able to see any of their previous responses. After giving each response, they were to look up at the experimenter to indicate that they had done so.

The strategy condition was similar to the random condition, except that the subjects were told to make their choices in such a manner that if another person were trying to predict which number would be selected next, he or she would not be able to do so. In this case, then, the emphasis was placed on trying to generate an unpredictable sequence of numbers, with no mention being made of the term "random."

Otherwise, the procedures for the two conditions were identical. To control approximately the rate at which the subjects generated the numbers, the experimenter announced at 4-sec intervals that another number was to be selected. A brief practice session of 10 trials preceded the 50 experimental trials so that the subjects could learn to pace their selections accordingly. The generation rate was sufficiently slow that none of the subjects had difficulty keeping up with it. At the conclusion of the experiment, they were debriefed, and their response cards were checked for completeness before their data were analyzed. 
Table 1

Mean Frequency of Numbers Generated (in Percent) in Each Condition

\begin{tabular}{lcccc}
\hline & \multicolumn{4}{c}{ Number } \\
\cline { 2 - 5 } Condition & 1 & 2 & 3 & 4 \\
\hline Random & 25.5 & 25.4 & 25.3 & 23.8 \\
Strategy & 23.6 & 25.1 & 27.1 & 24.2 \\
Expected & 25.0 & 25.0 & 25.0 & 25.0 \\
\hline
\end{tabular}

Table 2

Mean Frequency of Repetitions of Same Number (in Percent) in Each Condition

\begin{tabular}{lccc}
\hline & \multicolumn{3}{c}{ Repetitions of Same Number } \\
\cline { 2 - 4 } Condition & 1 & \multicolumn{1}{c}{2} & \multicolumn{1}{c}{3} \\
\hline Random & 5.36 & .24 & .08 \\
Strategy & 11.68 & 1.76 & .40 \\
Expected & 18.74 & 4.70 & 1.16 \\
\hline
\end{tabular}

\section{RESULTS}

As shown in Table 1, the mean frequencies with which each of the four numbers was generated by the subjects in the two conditions did not differ $(F<1)$. The only effect suggested by this first-order analysis was a marginally significant main effect of number on frequency $[F(3,144)=2.31, p<.08]$, meaning that there was a tendency for the middle two numbers in the set to be selected, on the average, slightly more often than the outer two numbers. Nevertheless, the mean generation frequencies for each number corresponded reasonably well to the base rate of $25 \%$ expected by chance.

Table 2 presents the mean frequency of repetitions of the same number in each condition. For this measure, the two groups clearly differed. The subjects in the strategy condition generated significantly more number repetitions than did those in the random condition $[F(1,48)=36.56, p<.001]$. As Table 2 also shows, their average frequencies of single, double, and triple consecutive repetitions were significantly closer to the frequencies expected by chance $[F(2,96)=16.99$, $p<.001]$. The main effect of the length of repetitions was also significant $[\mathrm{F}(2,96)=141.24, \mathrm{p}<.001]$, indicating that, on the average, shorter repetitions of a number were more common than were longer repetitions.

\section{DISCUSSION}

This study provides a demonstration that instructing people to try to behave in an unpredictable manner can lead to performance that, in certain respects, more closely approximates randomness than that produced when people are specifically told to try to behave in a random manner. The likely explanation for this difference is, as suggested by Kahneman and Tversky (1972; see also Tversky \& Kahneman, 1974), that people often believe that, in a random sequence, no local structure or order should exist, since such accidental regularities are not representative of the population as a whole. They therefore attempt to avoid repetitions of the same response when they try to behave randomly, which unwittingly results in departures from randomness. These departures can be avoided, to some degree, by strategically attempting to be unpredictable, such that another person would not be able to guess what their next response would be. Apparently, the application of this strategy makes closer contact with the intuition that sometimes an immediate repetition of a response is not to be expected than does the application of the representativeness heuristic.

Although the findings presented in this report suggest one way in which a person might more effectively introduce randomness into his or her behavior, there are certain to be intrinsic limitations on the extent to which this can be accomplished in practice. For example, there will always be constraints on how accurately previous responses can be remembered and then considered in determining the next response, so that behavior may never be fully unpredictable, despite efforts to the contrary (see, in particular, the discussion of this issue by Wagenaar, 1972). As a result, attempts to be elusive in planning behavior can be only partially successful, at least without the assistance of random-number tables or similar external aids.

\section{REFERENCES}

GARDNER, M. (1981). Science: Good, bad, and bogus. Buffalo, NY: Prometheus Books.

Kahneman, D., \& Tversky, A. (1972). Subjective probability: A judgment of representativeness. Cognitive Psychology, 3, 430-454.

LOPEs, L. L. (1982). Doing the impossible: A note on induction and the experience of randomness. Journal of Experimental Psychology: Learning, Memory, and Cognition, 8, 626-636.

Tversky, A., \& Kahneman, D. (1974). Judgment under uncertainty: Heuristics and biases. Science, 185, 1124-1131.

WAgenaAR, W. A. (1972). Generation of random sequences by human subjects: A critical survey of literature. Psychological Bulletin, 77, 65-72.

\section{NOTE}

1. The formal problems of how to define precisely what constitutes a random sequence of events, and of how to detect subtle departures from randomness, are complex and beyond the scope of this report. For a recent discussion, see Lopes (1982).

(Manuscript received for publication October 10, 1983.) 